\title{
Recanalization of the left pulmonary artery with radio-frequency perforation and stent angioplasty after failed intraoperative stent implant.
}

\author{
Sebastian Prida, Matthew D. Brown, John P. Breinholt* \\ Department of Pediatrics, University of Texas Health Science Center, Houston, Texas, United States
}

\begin{abstract}
Patients with pulmonary atresia with a ventricular septal defect are often palliated with a right ventricle to pulmonary artery conduit. Pulmonary artery interventions can result in the development of stenosis, and less commonly, atresia. We describe a novel case where an 11-month-old infant male with pulmonary atresia, ventricular septal defect, and bilateral branch pulmonary artery stenosis developed discontinuity of the left pulmonary artery, and was successfully recanalized with radiofrequency perforation and stent angioplasty, after a failed attempt at an intraoperative stent implantation.
\end{abstract}

Keywords: Congenital heart disease, Pulmonary angiography, Pediatric intervention, Congenital heart surgery.

Accepted on 15 March, 2018

\section{Introduction}

Patients with tetralogy of Fallot or pulmonary atresia with a ventricular septal defect (VSD) are often palliated with a modified Blalock-Taussig (BT) shunt, prior to undergoing definitive repair later in infancy. Pulmonary artery distortions are commonly seen after placement of BT shunts as a result of tenting, local scar formation, or vessel compression [1]. Despite the use of anticoagulant therapy, acute occlusion of segments of the pulmonary anastomoses can occur [2].

Transcatheter recanalization and reconstruction of complete, longstanding vessel occlusion using radiofrequency (RF) current perforation, with subsequent balloon dilatation and stent implantation, has been used for recanalization of atretic vessels in the pulmonary circulation $[1,3]$. We describe the recanalization of an occluded left pulmonary artery (LPA) with $\mathrm{RF}$ perforation and subsequent stent angioplasty after failed hybrid stent angioplasty.

\section{Case Report}

An infant male with a history of pulmonary atresia, VSD, right aortic arch, and bilateral branch pulmonary artery stenosis underwent placement of a right-sided modified BT shunt at 10 days of age.

At 9 months of age, the patient presented for complete repair, including VSD closure and placement of a right ventricle to pulmonary artery (RV-PA) conduit. During the procedure, the right pulmonary artery (RPA) appeared stenotic and underwent patch augmentation. The complete repair included VSD closure, placement of a $13 \mathrm{~mm}$ aortic homograft RV-PA conduit, RPA reconstruction, creation of atrial septal defect, and ligation and division of the BT shunt. Oxygen saturation after surgery was greater than $80 \%$.

During outpatient follow-up, there was increasing concern about flow into the LPA and he was referred for catheterization at 11 months of age, where he was found to have discontinuity of the LPA. An extensive effort was made to enter the LPA, including use of multiple catheters and wires, however, the occlusion had occurred at the LPA branch point, and a long sheath or catheter could not be stabilized at the site for more aggressive probing (Figure 1).

It was also determined that RF perforation would not be sufficiently controlled due to this positional instability. The effort at that point was aborted. The periprocedural echocardiogram demonstrated a moderately enlarged right atrium and ventricle with normal systolic function. The LPA was not visualized, and the Doppler gradient to the RPA was 52 $\mathrm{mmHg}$. There was mild pulmonary insufficiency.

The patient was referred to surgery to attempt open LPA recanalization, and stent placement as a hybrid procedure. During surgery, the main pulmonary artery was opened and the area probed to encounter the LPA tract. A probe entered a space thought to be the LPA, and was progressively dilated to $3.5 \mathrm{~mm}$ with a Hegar dilator. At this stage, it was decided to intraoperatively place a $16 \mathrm{~mm}$ EV3 Intrastent Max LD biliary stent (Medtronic, Minneapolis, MN) mounted on a Z-Med II 8 $\times 20 \mathrm{~mm}$ balloon (Numed, Inc., Hopkinton, NY). The procedure was limited by a portable fluoroscopy machine without recording capabilities. A 0.035 " wire was extended into the lumen, followed by the balloon/stent apparatus. The stent was deployed under direct visualization. Angiography did not reveal flow into the LPA, but demonstrated a small tract distal to the stent that did not appear intraluminal. Its appearance was similar to the angiogram taken at the subsequent catheterization (Figure 2A). Given these findings, the procedure was terminated, the stent left in place, and the child was recovered in the pediatric ICU.

Six days later, he was taken to the cardiac catheterization laboratory where simultaneous angiograms in the LPA stent and a left pulmonary vein wedge (LPVW) injection delineated the relationship of the new stent tract and the native LPA (Figure 2B). 
Citation: Prida S, Brown MD, Breinholt JP. Recanalization of the left pulmonary artery with radio-frequency perforation and stent angioplasty after failed intraoperative stent implant. J Invasive Noninvasive Cardiol. 2018;1(1):14-17.

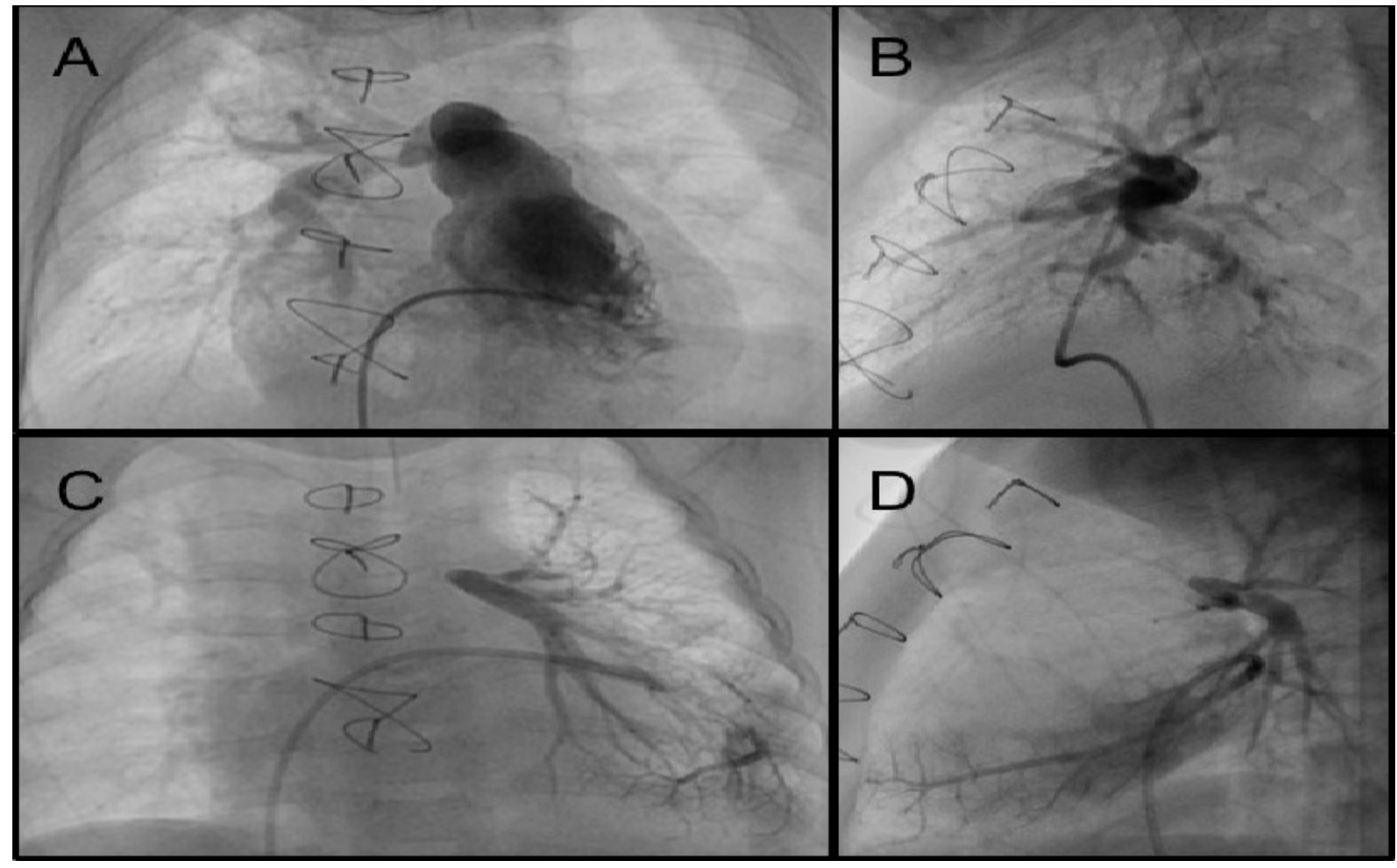

Figure 1. A) Anteroposterior (AP) projection angiogram in the right ventricle. The right ventricular outflow is unobstructed, the RPA is well visualized, and no pulmonary artery branching to the left lung is visualized. B) Lateral projection (60 ${ }^{\circ}$ LAO) demonstrates the location of LPA atresia with no significant length of LPA (white arrow) in which to place, and stabilize, a catheter. C) AP projection showing left pulmonary vein wedge $(L P V W)$ with retrograde filling of the native LPA. There is no connection to the main pulmonary artery. D) Lateral projection of the LPVW angiogram.

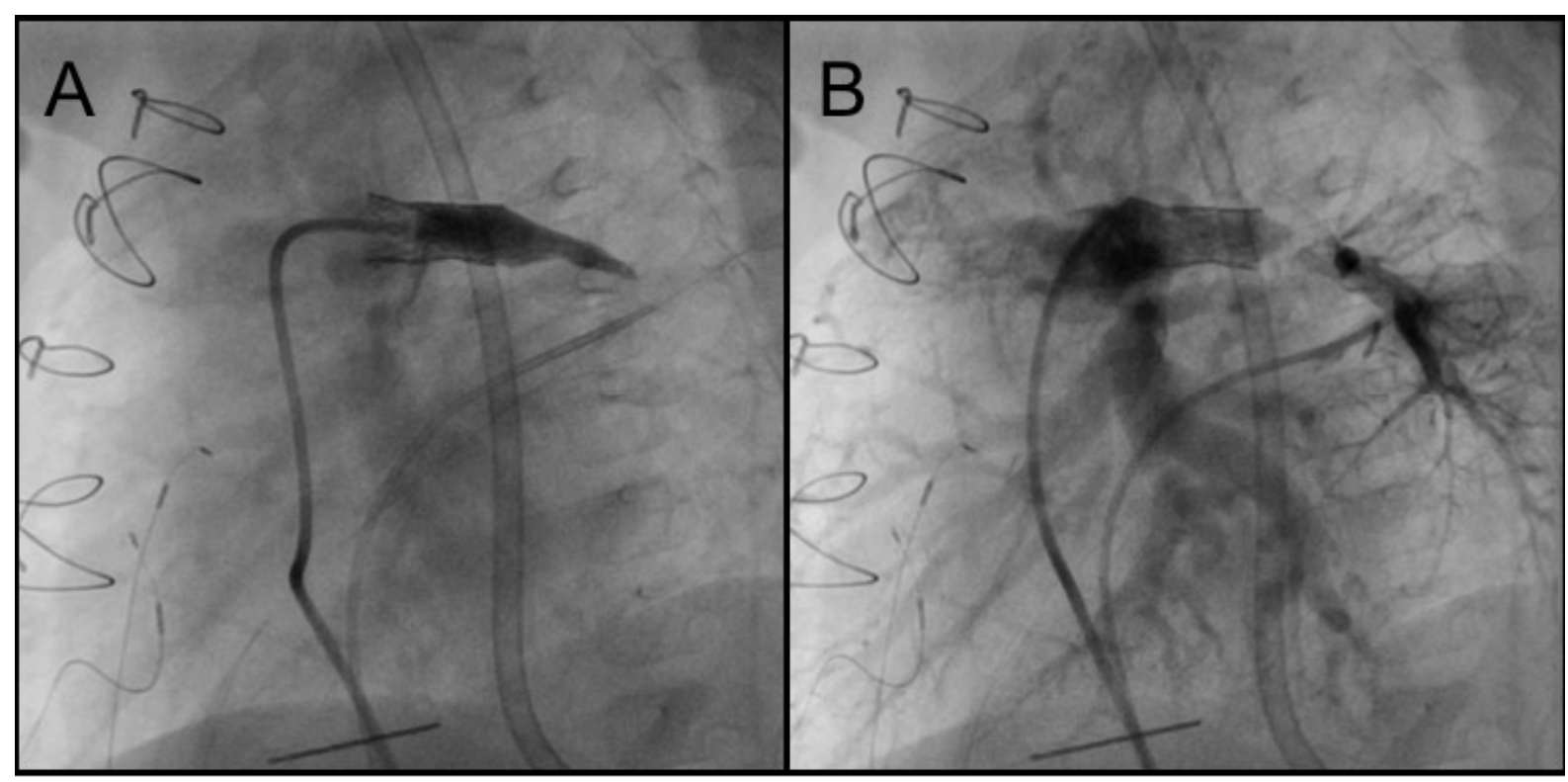

Figure 2. A) Lateral projection $\left(60^{\circ}\right.$ LAO) angiogram displaying contrast within the surgically placed stent, ending in a blind tract. B) Simultaneous angiography in the LPA stent and a left pulmonary vein wedge angiogram. The surgical tract is superior to the native LPA and discontinuous from the native vessel.

A Baylis RF wire and microcatheter system (Baylis Medical, Montreal, QC) were advanced through a 4-French JR3 catheter that had been placed in the EV3 stent. Seven watts was applied for 2 seconds and the wire crossed into the LPA (Figure 3A). The microcatheter was then advanced and an angiogram was performed to verify catheter position (Figure 3B). 


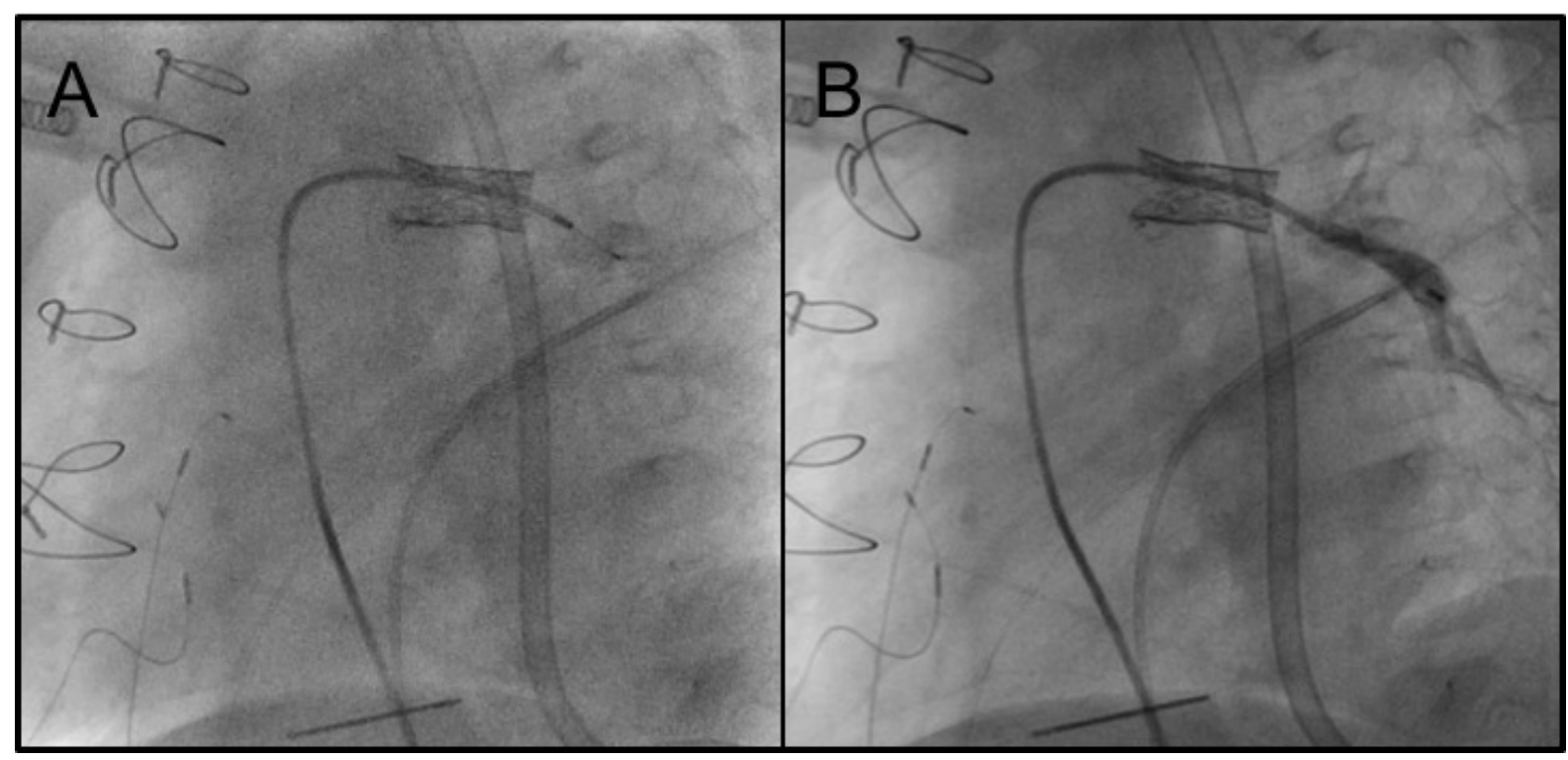

Figure 3. A) Lateral projection angiogram $\left(60^{\circ} \mathrm{LAO}\right)$ of $R F$ perforation. The $R F$ wire has been advanced into the native LPA. B) Lateral projection angiogram through the microcatheter that verifies proper entry of the wire in the pulmonary vascular.

Thereafter, the RF wire was exchanged for a V-18 wire (Boston Scientific, Canton, MA). We then advanced and deployed a Palmaz Blue $7 \times 15 \mathrm{~mm}$ stent (Cordis, Milpitas, CA), overlapping with the MAX LD stent, resulting in patency of the LPA from the RV-PA conduit, with no extravasation of contrast (Figure 4).

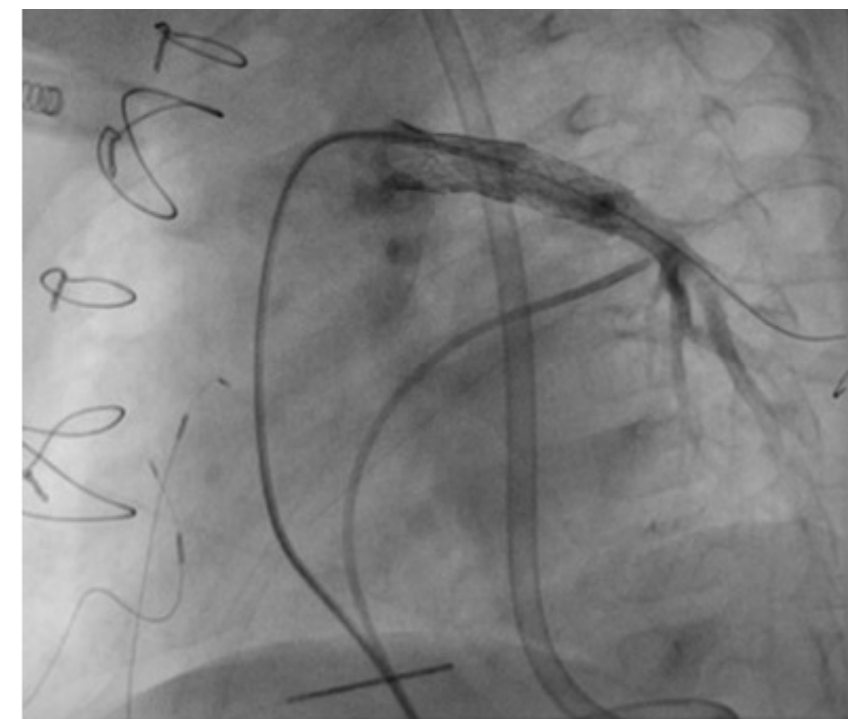

Figure 4. Lateral projection angiogram $\left(60^{\circ} \mathrm{LAO}\right)$ showing the final result with wide patency of the LPA.

Right ventricular pressure was decreased from $56 \mathrm{mmHg}$ to 44 $\mathrm{mmHg}$, and the gradient from the main pulmonary artery to the LPA was $6 \mathrm{mmHg}$. Oxygen saturations increased from the mid-80's on 1-3 L of nasal cannula oxygen the day before the procedure to mid- to high-90's on room air the day following the procedure. The echocardiogram the day following the procedure demonstrated good flow across the LPA stent with a peak Doppler gradient of $16 \mathrm{mmHg}$. A prior RPA stent implant had been dilated and the peak Doppler gradient was now 27 $\mathrm{mmHg}$. There was moderate pulmonary insufficiency.
One year later, the patient had a repeat catheterization due to an increased gradient noted to the bilateral branch pulmonary arteries. There was a $34 \mathrm{mmHg}$ gradient from the conduit to the LPA. After angioplasty with an $8 \times 20 \mathrm{~mm}$ Sterling balloon (Boston Scientific, Canton, MA), the gradient decreased to 11 $\mathrm{mmHg}$.

\section{Discussion}

RF perforation of an atretic segment, followed by balloon dilation and stent implantation to regain vessel continuity in the pulmonary circulation is feasible even in the early postoperative period [1,4]. A previous report describes successful recanalization of an occluded LPA in a 7-year-old child using transcatheter radiofrequency perforation, followed by balloon dilation and subsequent stent implantation [3]. Another describes an 18-month-old infant with a discrete atretic segment of the RPA in which a similar technique was employed with an RF catheter, balloon dilation and stent implantation [1]. A third case describes the first use of the Baylis RF perforation wire to reestablish LPA continuity in a 14-month-old infant who had previously undergone repair of pulmonary atresia [5].

In our case, the attempted surgical intervention with open, intraoperative LPA stent placement failed due to creation of a false tract. The surgery was abandoned due to concern of potential bleeding complications by attempting to surgically repair the connection between the main pulmonary artery and the LPA. However, the retained stent allowed a subsequent catheterization where a catheter could be placed in a stable position within the stent tract to allow use of the Baylis RF wire system. As such, we successfully recanalized the LPA with the aid of an additional stent to bridge from the intraoperative stent to the native LPA.

After loss of continuity of the LPA and a failed attempt at a hybrid procedure with less than ideal imaging capabilities, we 
Citation: Prida S, Brown MD, Breinholt JP. Recanalization of the left pulmonary artery with radio-frequency perforation and stent angioplasty after failed intraoperative stent implant. J Invasive Noninvasive Cardiol. 2018;1(1):14- 17.

were able to salvage the LPA with the assistance of RF perforation and subsequent stent angioplasty. Indeed, the surgically implanted stent became a crucial aid to allow stable catheter and wire position and thus enable successful recanalization of the LPA and placement of a stent.

\section{Conclusion}

To our knowledge, this is a novel description of utilizing an intraoperatively placed stent, albeit in a false tract, that allowed successful recanalization of an occluded LPA with RF perforation and stent angioplasty.

\section{References}

1. Pedra CA, Filho RM, Arrieta RS, et al. Recanalization of a discrete atretic right pulmonary artery segment with a new radiofrequency system. Catheter Cardiovasc Interv. 2003;60:82-7.

2. Sreeram N, Emmel M, Trieschmann U, et al. Reopening acutely occluded cavopulmonary connections in infants and children. Interact Cardiovasc Thorac Surg. 2009; 10:383-8.

3. Fink C, Peuster M, Bertram H, et al. Transcatheter recanalization of the left main pulmonary artery after four years of complete occlusion. Catheter Cardiovasc Interv. 2001;53:81-4.

4. Qureshi AM, Hill JA, Prieto LR, et al. Transcatheter Recanalization of Totally Occluded Proximal Pulmonary Arteries and Major Systemic Veins in Patients With Congenital Heart Disease. Am J Cardiol. 2013;111:412-7.

5. Kort HW, Balzer DT. Radiofrequency perforation in the treatment of acquired left pulmonary artery atresia following repair of tetralogy of Fallot. Catheter Cardiovasc Interv. 2003;60:79-81.

\section{*Correspondence to:}

John P. Breinholt

Department of Pediatrics

University of Texas Health Science Center

6410 Fannin Street Suite 425, Houston, Texas, United States

Tel: +1-713-500-5737

E-mail: john.p.breinholt@uth.tmc.edu 